Research Article

\title{
Controlled Depolymerization of Cellulose Fibres Isolated from Lignocellulosic Biomass Wastes
}

\author{
Suh Cem Pang $(\mathbb{D}$, Lee Ken Voon, and Suk Fun Chin \\ Resource Chemistry Programme, Faculty of Resource Science \& Technology, Universiti Malaysia Sarawak, 94300 Kota Samarahan, \\ Sarawak, Malaysia \\ Correspondence should be addressed to Suh Cem Pang; scpang@unimas.my
}

Received 25 April 2018; Revised 30 May 2018; Accepted 5 June 2018; Published 19 July 2018

Academic Editor: Qinglin Wu

Copyright (C) 2018 Suh Cem Pang et al. This is an open access article distributed under the Creative Commons Attribution License, which permits unrestricted use, distribution, and reproduction in any medium, provided the original work is properly cited.

\begin{abstract}
Various types of lignocellulosic biomass wastes (LBW) had been successfully converted into cello-oligomers with different chain lengths via a controlled depolymerization process. Cellulose fibres isolated from LBW samples were dissolved with room temperature ionic liquid (RTIL) in the presence of an acid catalyst, Amberlyst 15 DRY. The effects of reaction time on the degree of polymerization and yields of water-insoluble cello-oligomers formed were studied. Besides, the yields of water-soluble cello-oligomers such as glucose and xylose were also determined. The depolymerization of cellulose fibres isolated from LBW was observed to follow both second-order and pseudo-second order kinetics under specific conditions. As such, cello-oligomers of controllable chain lengths could be obtained by adjusting the duration of depolymerization process under optimized conditions.
\end{abstract}

\section{Introduction}

In recent decades, the production of biofuels from renewable biomass have gained increasing attention of scientists worldwide [1]. However, the use of food resources such as glucose derived from direct extraction of saccharose in sugarcane and sugar beet or through hydrolysis of corn starch for production of biofuels would inevitably compete with food supply [2]. As such, the use of cellulose fibres derived from lignocellulosic biomass wastes (LBW) as the feedstock is considered to be more appropriate than starch and saccharose. Cellulose is the most abundant organic compound on earth, and utilization of cellulose fibres derived from LBW does not compete with food supply [3]. Nevertheless, the presence of extensive intra- and intermolecular hydrogen bond networks, the basicity of glycosidic bonds, and high crystallinity of cellulose have posed great challenges for breaking down cellulose fibres through both chemical or biological processes $[4,5]$.

In conventional hydrolysis of lignocellulosic biomss materials, mineral acids such as hydrochloric acid, sulfuric acid, or even hydrofluoric acid are being used as liquid catalysts [6]. These liquid catalysts need to be employed in high concentration and/or at high reaction temperature (170$240^{\circ} \mathrm{C}$ ) in order to enhance product yields. Typically, lignocellulosic raw materials are not being completely solubilized in the reaction medium [7]. Although valuable products are obtained from the hydrolysis process, full-scale hydrolyses of cellulose have never been commercially implemented due to issues which include corrosion, high energy demand, catalyst recovery, and degradation of sugars.

In recent years, ionic liquids have been used as solvents and catalysts in various fields of research $[8,9]$. Ionic liquids are defined as salts with a melting point below $100^{\circ} \mathrm{C}$ which exhibit several unique properties such as negligible vapor pressure, extraordinary dissolution properties, wide liquid range, high electrical conductivity, low volatility, and low flammability $[10,11]$. Ionic liquids have been reported to dissolve cellulose for up to $25 \mathrm{wt} \%$ [12]. This has led to intense research into the conversion of biomass in ionic liquids. Besides, ionic liquids can be easily recovered and recycled by evaporating water or alcoholic residues using vacuum distillation and then purified by passing it through a neutral alumina column $[5,13]$. All these unique properties of ionic liquid have led to their uses as the preferred solvent for the depolymerization of cellulose. 
The depolymerization of cellulose in ionic liquid occurs by the addition of hydronium ion $\left(\mathrm{H}_{3} \mathrm{O}^{+}\right)$species, which cleavage the $1,4 \beta$-glycosidic bonds to form either cellooligomers or glucose during the initial stage. However, the presence of $\mathrm{H}_{3} \mathrm{O}^{+}$ions can further dehydrate glucose to produce 5-hydroxymethylfurfural, levulinic, and formic acids. These side products are prone to undergo aldol condensation with glucose to form humins which are difficult to be separated from useful products. Such reactions would reduce the efficiency of producing usable feedstock from lignocellulosic biomass. Therefore, it is important to control the depolymerization process such that reaction could be stopped at early stages in order to generate cello-oligomers instead of glucose and to avoid the formation of dehydration and polymeric by-products. However, the production of humins could not be avoided when mineral acid was being used as the catalyst during the hydrolysis of cellulose which occurred instantaneously [14].

Rinaldi and coworkers have reported the use of solid acids such as Amberlyst 15, Amberlyst 35, and Amberlyst 70 , as well as Nafion, sulfated zirconate, and zeolite Y as catalysts for the hydrolysis of cellulose dissolved in ionic liquid [15]. The macroreticulated resins functionalized with sulfonic groups (Amberlyst 15, Amberlyst 35) showed good catalytic activity for the depolymerization of cellulose fibres in an ionic liquid. Amberlyst acid resins are highly stable in acidic anion ionic liquid as compared to other basic anion ionic liquids such as 1-butyl-3-methylimidazolium acetate, which leads to rapid decomposition of the catalyst [15]. During the depolymerization of cellulose in ionic liquid, $\mathrm{H}_{3} \mathrm{O}^{+}$ ions are being progressively released from Amberlyst 15 into ionic liquid within the first hour of contact between cellulose solution and solid catalyst, resulting in an induction period for the formation of glucose [5]. This shows that the depolymerization of cellulose solubilized in ionic liquid in the presence of Amberlyst 15 could afford control of the hydrolysis process during the early stage of cello-oligomer production. However, the study on controlling chain lengths of cellooligomers is still very lacking. Understanding the mechanisms of the depolymerization process is essential for enabling the production of cello-oligomers with different chain lengths which are suitable for conversion to biofuels or methylcellulose as well as platform chemicals such as surfactants, thickening agents, and glues and as rheology modifiers [16].

Herein, we have reported our findings on controlled depolymerization of cellulose fibres which were isolated from four different types of lignocellulosic biomass wastes including sawdust from wood of Acacia magnium, sugarcane bagasse, corn cob, and pith waste of sago (Metroxyln sagu). Cellulose fibres were depolymerised by dissolving in ionic liquid in the presence of an acid catalyst, Amberlyst 15 DRY. The yields of cello-oligomers, the mean degree of polymerization (DPw), and the distribution of $\mathrm{DPw}$ as well as the major side products dervied from various cellulose fibres of various lignocellulosic biomass wastes were determined. Besides, the kinetics of the depolymerization process of cellulose fibres under specific conditions were investigated.

\section{Experiment}

2.1. Materials. Four different types of LBW materials were used in this study including wood sawdust, sugarcane bagasse, corncob, and sago pith wastes. The sawdust of wood (Acacia mangium) was obtained from the wood research lab of Univerisiti Malaysia Sarawak. Sugarcane bagasse (Saccharum officinarum spp.) and corncob (Earlivee sweet corn) were collected from a local market, whereas the pith waste of sago (Metroxylon sagu) was collected from a local sago starch-processing factory. All lignocellulosic biomass waste samples were washed with deionized water to remove impurities such as sand and dirt, dried in an oven, and than finely ground using a grinder. Sodium hydroxide $(\mathrm{NaOH})$, sodium chlorite $\left(\mathrm{NaClO}_{2}\right)$, acetic acid, silver nitrate $\left(\mathrm{AgNO}_{3}\right)$, methanol, and pyridine were purchased from Merck whereas 1methylimidazole, allyl chloride, dimethyl sulfoxide (DMSO), phenylisocyanate, tetrahydrofuran (THF), and Amberlyst 15 DRY were purchased from Sigma-Aldrich. Potassium sodium tartrate and phenol were purchased from JT Baker. Ultrapurewater $\left(\sim 18.2 \mathrm{M} \Omega \cdot \mathrm{cm}, 25^{\circ} \mathrm{C}\right)$ was obtained from the Water Purifying System (ELGA Model Ultra Genetic). All chemicals were used without further purification.

\subsection{Extraction of Cellulose Fibres from Lignocellulosic} Biomass Wastes. Cellulose fibres were isolated from various lignocellulose biomass wastes based on the method reported by Sun and Cheng [17]. Samples of sawdust, sugarcane bagasse, and corn cob and sago pith waste were first treated with $\mathrm{NaOH}(12 \mathrm{wt} \%)$ at room temperature under stirring (1000 rpm) for $8 \mathrm{~h}$ in order to remove the hemicellulose. This procedure was repeated twice. The $\mathrm{NaOH}$ treatment had also removed starch granules contained in sago pith waste samples. Iodine test was performed to confirm total removal of starch residues in sago pith waste. In addition, the $\mathrm{NaOH}$-treated samples were delignified in a $\mathrm{NaClO}_{2}$ solution ( $1 \mathrm{wt} \%$ ) which had been adjusted to $\mathrm{pH} 3.58$ with acetic acid (10 wt\%) and maintained at $70^{\circ} \mathrm{C}$ for $5 \mathrm{~h}$. Cellulose fibres obtained were washed with deionized water and dried in an oven at $80^{\circ} \mathrm{C}$ until constant weight.

2.3. Synthesis of 1-Allyl-3-methylimidazolium Chloride (AMIMCl). The room temperature ionic liquid, AMIMCl, was synthesized based on the procedure reported by Zhang et al. with some modifications [18]. 1-Methylimidazole was placed into a three-neck round-bottom flask, and then allyl chloride was added dropwise to the flask at room temperature in the volume ratio of $1: 1.2(v / v \%)$. The reaction mixture was stirred magnetically under reflux at about $55^{\circ} \mathrm{C}$ for $7 \mathrm{~h}$. The residual allyl chloride was eliminated using a rotary evaporator, whereas the unreacted 1-methylimidazole was removed by washing thrice with an excess amount of ether. The resulting ionic liquid was stored with a $3 \mathrm{~A}$-size molecular sieve in a vacuum desiccator.

2.4. Depolymerization of Cellulose Fibres. A measured volume of ionic liquid, AMIMCl $(10 \mathrm{~mL})$, was preheated at $80^{\circ} \mathrm{C}$ for $30 \mathrm{~min}$ to make it less viscous. A sample of cellulose fibres $(0.50 \mathrm{~g})$ was then added into the ionic liquid and magnetically stirred until it was completely dissolved. $100 \mu \mathrm{L}$ of deionized 
water was added into cellulose solution and stirred for $2 \mathrm{~min}$. Subsequently, the Amberlyst $15 \mathrm{DRY}$, an acid catalyst $(0.05 \mathrm{~g}$, $0.23 \mathrm{mmol} \mathrm{H}_{3} \mathrm{O}^{+} \cdot \mathrm{g}^{-1}$ ), was added. After the predetermined reaction durations, the solid catalyst was separated by filtering with a 20-mesh sieve and cello-oligomers formed were precipitated by adding water dropwise into the reaction medium. The cello-oligomers were separated into solid and liquid fractions by centrifugation at $6000 \mathrm{rpm}$. The solid fraction of cello-oligomer samples was washed with ultrapure water until no chloride ions were detected using $0.1 \mathrm{M}$ silver nitrate solution and dried at $60^{\circ} \mathrm{C}$ in an oven until constant weight and stored in a vacuum desiccator. The liquid fractions of dissovled cello-oligomers were collected separately and stored at $4^{\circ} \mathrm{C}$.

2.5. Determination of Degree of Polymerization (DPw). The mean and distribution of DPw of cello-oligomers were determined based on the method reported by Kačík et al. [19]. Generally, phenylisocyanate $(1 \mathrm{~mL})$ was added to a cellulose sample $(2 \mathrm{mg})$ dispersed in dry pyridine $(6 \mathrm{~mL})$, and the resulting mixture was allowed to react at $110^{\circ} \mathrm{C}$ for $6 \mathrm{~h}$. Upon completion of reaction, excess phenylisocyanate was decomposed by adding methanol $(2 \mathrm{~mL})$. The resulting clear yellow solution was then precipitated in methanol under vigorous stirring. The precipitate was washed with acetic acid $(10 \mathrm{wt} \%)$ to remove pyridine and then washed with methanol (70 wt\%). Finally, the cellulose tricarbanilate (CTC) samples obtained were dried at $60^{\circ} \mathrm{C}$ in an oven until constant weight. CTC samples were then dissolved in tetrahydrofuran $(2 \mathrm{mg} / \mathrm{mL})$ and analyzed by gel permeation chromatography (GPC). Sample solutions were filtered through filters with a mean pore size of $0.20 \mathrm{~mm}$ (Whatman, Puradisc 25 NYL). The GPC analysis was performed using a HPLC (Perkin-Elmer FLEXAR) fitted with mixed-bed GPC columns (3 columns, PLgel $10 \mu \mathrm{M}$ MIXED B, Polymer Laboratories, $7.5 \mathrm{~mm} \mathrm{ID} \times 300.0 \mathrm{~mm}$ ) at $30^{\circ} \mathrm{C}$, and stabilized THF was used as the eluent $(1.0 \mathrm{~mL} / \mathrm{min})$. A UV-Vis detector was used for cello-oligomer detection at the wavelength of $240 \mathrm{~nm}$. Prior to GPC analysis, the system was calibrated using polystyrene standards (Agilent EasiVial, $1.62 \times 10^{2}$ to $\left.7 \times 10^{6} \mathrm{~g} / \mathrm{moL}\right)$. The mean DPw was calculated by dividing the molecular weight of CTC samples (W) by the monomer equivalent weight of CTC ((1)):

$$
\mathrm{DPw}=\frac{\mathrm{W}}{519} .
$$

2.6. Determination of Water-Soluble Oligomers. The total reducing sugar (TRS) was quantified using the 3,5-dinitrosalicylic acid (DNS) assay [20]. DNS reagent $(2.0 \mathrm{~mL})$ was added to the liquid fraction $(1.0 \mathrm{~mL})$ and heated in a boiling water bath for about $10 \mathrm{~min}$. The resulting solution was cooled to room temperature and then diluted with deionized water by a factor of 10 . The absorbance of total reducing sugar (TRS) was measured at the wavelenght of $540 \mathrm{~nm}$ using a UV-Vis spectrophotometer (Shimadzu Model UV-160A). The concentration of TRS was calculated from the calibration curve of standard glucose solutions.

Glucose and xylose were analysed using high-performance liquid chromatography (HPLC, Shimazu Model LC-20A) equipped with a refractive index detector. The chromatographic separations were carried out using a column (BioRad Aminex HPX-871, $100 \times 7.8 \mathrm{~mm}$ ) and diluted sulfuric acid $(5 \mathrm{mM})$ as the mobile phase. Samples were analysed in isocratic mode at a flow rate of $0.8 \mathrm{~mL} / \mathrm{min}$ at $65^{\circ} \mathrm{C}$ and $20 \mu \mathrm{L}$ injection volume. All detected compounds were identified by comparing retention times with those of corresponding standard reference compounds. Amounts of products formed were quantified by comparing with calibration curves of external authentic samples.

2.7. FTIR Analysis. The chemical structure of cellulose fibres was characterized by Fourier transform infrared spectrometry (FTIR) (Thermo Scientific Nicolet iS10). The dried and ground cellulose sample was mixed thoroughly with potassium bromine $(\mathrm{KBr})$ powder at a ratio of $1: 100(w / w)$, before being compressed into pellets. Prior to its use, the KBr powder was dried in an oven to ensure its water content was less than $1 \mathrm{wt} \%$. The FTIR spectrum was generated by scanning at a resolution of $4 \mathrm{~cm}^{-1}$ with a total of 64 scans for each sample within the wave number range of $4000-500 \mathrm{~cm}^{-1}$.

2.8. Depolymerization Kinetics of Cellulose Fibres. The depolymerization kinetics of cellulose fibres isolated from various lignocellulosic biomass wastes were investigated based on (2) and (3) $[21,22]$.

$$
\begin{gathered}
\text { Second-order : } \frac{1}{\mathrm{DPw}}=k t+\frac{1}{\mathrm{DPo}_{\mathrm{w}}}, \\
\text { Pseudo-second-order }: \frac{t}{\mathrm{DPw}}=\frac{1}{\mathrm{DPo}} t+\frac{1}{k(\mathrm{DPo})},
\end{gathered}
$$

where DPo is the mean degree of polymerization values of original cellulose fibres, DPw is the mean degree of polymerization values of cello-oligomers, $t$ is the reaction time of the depolymerization process, and $k$ is the reaction constant.

\section{Results and Discussion}

3.1. FTIR Spectra. All FTIR spectra showed similar characteristic absorption peaks as compared with those of standard pure cellulose (Figure 1). A strong broad peak observed at $3383-3412 \mathrm{~cm}^{-1}$ was attributed to O-H stretching of hydroxyl groups of cellulose with moisture [23]. The absorption bands between $2899 \mathrm{~cm}^{-1}$ and $2902 \mathrm{~cm}^{-1}$ were due to C-H stretching of methyl and methylene groups [24]. Absorption bands located at $1627-1651 \mathrm{~cm}^{-1}$ were due to adsorbed water molecules [25]. Appearances of weak absorption peaks at 1429$1433 \mathrm{~cm}^{-1}$ and $1317-1372 \mathrm{~cm}^{-1}$ were assigned to bending of $\mathrm{CH}_{2}$ cellulose [26]. Weak absorption peaks at $1162-$ $1164 \mathrm{~cm}^{-1}$ were due to $\mathrm{O}-\mathrm{H}$ bonds at $\mathrm{C} 2, \mathrm{C} 3$, and $\mathrm{C} 5$ of cellulose [25]. Strong peaks observed at about $1061 \mathrm{~cm}^{-1}$ were due to $\mathrm{C}-\mathrm{O}$ stretching [27]. The absorption peak observed at $897 \mathrm{~cm}^{-1}$ was due to $\beta$-glycosidic linkages between the sugar units [28].

Besides, FTIR spectra were used to confirm the removal of lignin and hemicelluloses from various LBW samples. After strong alkaline treatment, no absorption peak was observed at $1735 \mathrm{~cm}^{-1}$, which was attributed to the complete 


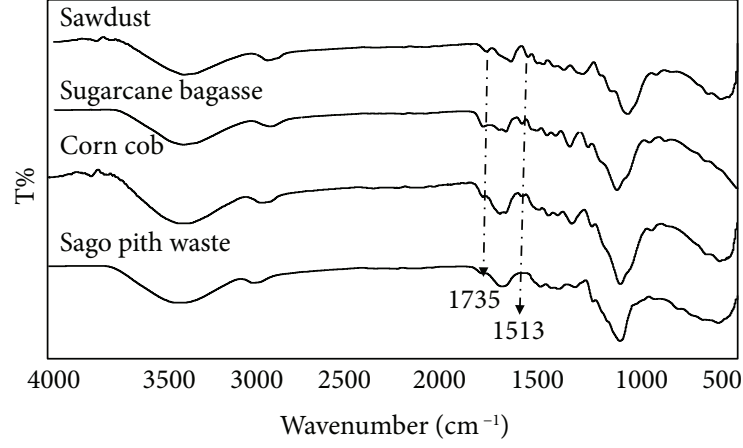

(a)

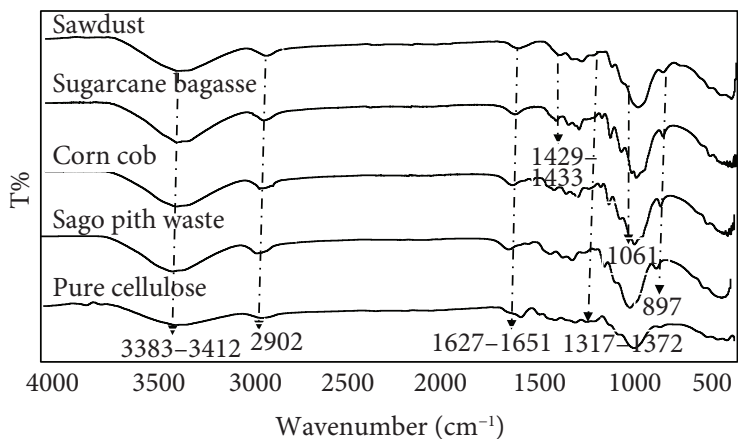

(b)

FIGURE 1: FTIR spectra of various lignocellulosic biomass waste samples (a) before and (b) after alkaline pretreament.

removal of hemicelluloses and/or lignin components [29]. In addition, no absorption peak attributed to the $-\mathrm{C}=\mathrm{C}-$ stretching of aromatic rings of lignin was observed at $1513 \mathrm{~cm}^{-1}$, thereby confirming that all lignin had been removed from cellulose fibres isolated from various LBW samples (Figure 1(b)) [30].

3.2. Depolymerization of Cellulose Fibres. Cellulose fibres isolated from sawdust, sugarcane bagasse, corn cob, and sago pith waste samples were depolymerized by dissolving in AMIMCl to form $5 \mathrm{wt} \%$ of cellulose solution and the subsequent addition of $0.05 \mathrm{~g}$ Amberlyst $15 \mathrm{DRY}(0.23 \mathrm{mmol}$ $\mathrm{H}_{3} \mathrm{O}^{+}$) as the catalyst. The schematic reaction mechanism of the depolymerization process is as shown in Figure 2. In this reaction, ion-exchange process occurred between the ionic liquid and acid catalyst resin, resulting in the release of $\mathrm{H}_{3} \mathrm{O}^{+}$species which cleavaged the $\beta$-glycosidic bonds in the cellulose chains to produce cello-oligomers and other side products such as reducing sugar, glucose, and xylose [5].

Figure 3 shows the mean DPw of cello-oligomers derived from cellulose fibres of various lignocellulosic biomass wastes at different reaction times $(5-120 \mathrm{~min})$. The mean $\mathrm{DPw}$ values of cello-oligomers were observed to decrease rapidly initially and then reached a constant value after reaction durations of 40 to $80 \mathrm{~min}$.

However, the distribution of DPw values or chain lengths of cello-oligomers produced were observed to be affected by initial DPw values of cellulose fibres used in the depolymerization process. Cellulose fibres derived from sawdust and corn cob with initial DPw values of about 350 were observed to produce cello-oligomers with mean DPw values of 82 and 118 , respectively, after a reaction time of about $40 \mathrm{~min}$ (Figure 3). However, cellulose fibres derived from sago pith waste and sugarcane bagasse with initial mean DPw values of 564 and 571, respectively, produced cello-oligomers with substantially lower DPw value of 30 and 35 after reaction times of up to $60 \mathrm{~min}$ and $100 \mathrm{~min}$, respectively. Cellulose fibres of sago pith waste exhibited a faster rate of depolymerization as compared with that of sugarcane bagasse which could be due to cellulose fibres with comparatively more uniform of DPw distribution.
3.3. Composition of Water-Soluble Oligomers. Generally, the yields of regenerated cello-oligomers obtained via the depolymerization of cellulose fibres of various LBW were observed to decrease with increasing reaction time due to the formation of water-soluble oligomers such as reducing sugar, glucose, and xylose (Figure 4). The major component of water-soluble oligomers produced from different types of cellulose fibres was reducing sugar. Yields of regenerated cello-oligomers and water-soluble oligomers derived from cellulose fibres of sawdust and corn cob after depolymerization for $120 \mathrm{~min}$ were $54.3 \mathrm{wt} \%$ and $6.15 \mathrm{wt} \%$, and 63.4 $\mathrm{wt} \%$ and $11.34 \mathrm{wt} \%$, respectively. However, yields of regenerated cello-oligomers from cellulose fibres of sugarcane bagasse and sago pith wastes after depolymerization for 120 min were comparatively lower at $30 \mathrm{wt} \%$ and $40 \mathrm{wt} \%$, respectively. Such lower yields could be attributed to comparatively higher percentages of water-soluble oligomers being produced (Figures 4(b) and 4(d)). In addition, cellulose fibres of sugarcane bagasse and sago pith wastes were being depolymerized to cello-oligomers with lower mean DPw values between 30 and 40, respectively (Figure 3). Probably, some shorter-chain cello-oligomers $(\mathrm{DPw}<10)$ were not precipitated, thereby resulting in the comparatively lower yields of cello-oligomers.

3.4. DPw Distribution of Cello-Oligomers. The DPw distribution of cello-oligomers obtained from cellulose fibres of various lignocellulosic biomasses was investigated by gel permeation chromatography (GPC) as shown in Figure 5. All cello-oligomers were observed to exhibit unimodal distribution of $\mathrm{DPw}$ at various predetermined reaction durations. Generally, the DPw distribution of cello-oligomers showed a gradual and continuous shift to lower mean DPw values with longer reaction time, indicating that the depolymerization process had caused a chain scission of cellulose molecules, thereby leading to a decrease in mean DPw values with increasing duration of depolymerization. Besides, the width of DPw distribution curves as indicated by the polydispersity index (PDI) was observed to decrease rapidly or gradually, indicating continual reduction in the polydispersity of cello-oligomers with the extent of the depolymerization process (Figure 5(e)). 


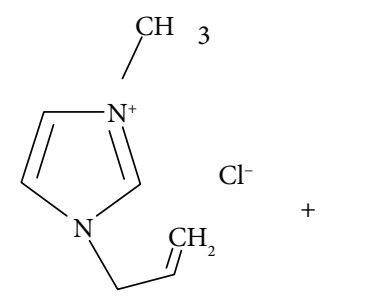<smiles>CCC(C)c1ccccc1</smiles>

AMIMCI

Amberlyst 15 DRY

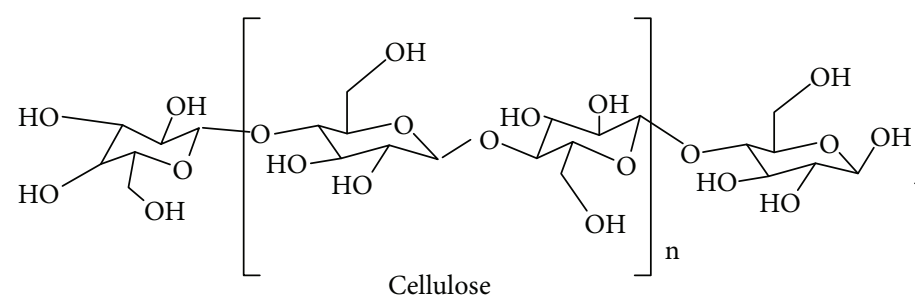

Cellulose

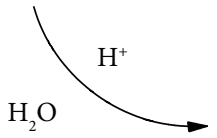<smiles>OC[C@H](O)[C@H](O)C(O)O</smiles>

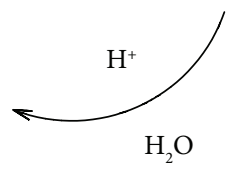

Glucose<smiles>OC1COC(O)C(O)C(O)C1O</smiles>

Xylose

FIGURE 2: Schematic reaction mechanism for the depolymerization process of cellulose fibres.

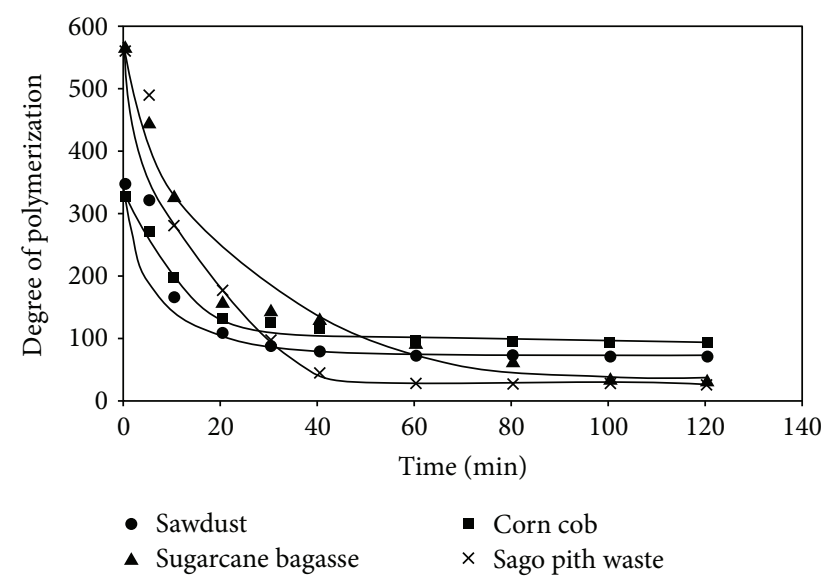

FIGURE 3: Degree of polymerization (DPw) of cello-oligomers derived from cellulose fibres of various lignocellulosic biomass at different reaction times: $(\bullet)$ sawdust $(\mathrm{DPw}=351),(\boldsymbol{\Delta})$ sugarcane bagasse $(\mathrm{DPw}=571),(\mathbf{\square})$ corn cob $(\mathrm{DPw}=329)$, and $(\mathbf{x})$ sago pith wastes $(\mathrm{DPw}=564)$. Parentheses show the initial $\mathrm{DPw}$ values of lignocellulosic biomass samples.
Cello-oligomers derived from cellulose fibres of sawdust were observed to exhibit substantially narrower DPw distribution with its mean peak shifted to lower DPw values after initial depolymerzation duration of $40 \mathrm{~min}$ (Figure 5(a)). However, peaks of the mean DPw distribution were observed to have shifted only slightly upon subsequent depolymerization durations between 40 and $120 \mathrm{~min}$. The PDI of cellooligomers formed was observed to decrease rapidly from 25.5 during the initial $40 \mathrm{~min}$ and thereafter decreased very gradually to 14.70 after depolymerization for $120 \mathrm{~min}$.

Similarly, cello-oligomers formed from cellulose fibres of sugarcane bagasse and corn cob samples showed a continual shift in their DPw peaks throughout the depolymerization duration of $120 \mathrm{~min}$ (Figures 5(b) and 5(c)). DPw distributions became progressively narrower with increasing depolymerization duration from $10 \mathrm{~min}$ to $120 \mathrm{~min}$ However, DPI values of cello-oligomers for both sugarcane bagasse and corn cob samples were observed to decrease gradually from 17.6 and 15.3 to 9.78 and 3.21, respectively, after depolymerization for 120 min (Figures 5(e)).

In the case of cello-oligomers formed from cellulose fibres of sago pith waste, DPw peaks were observed to 


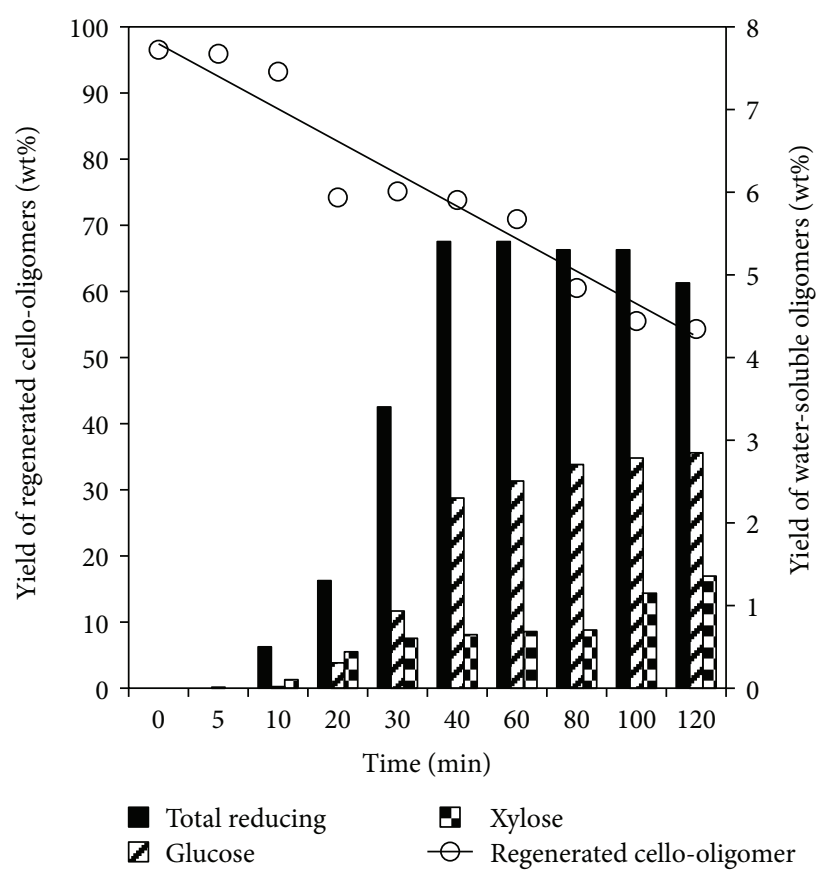

(a)

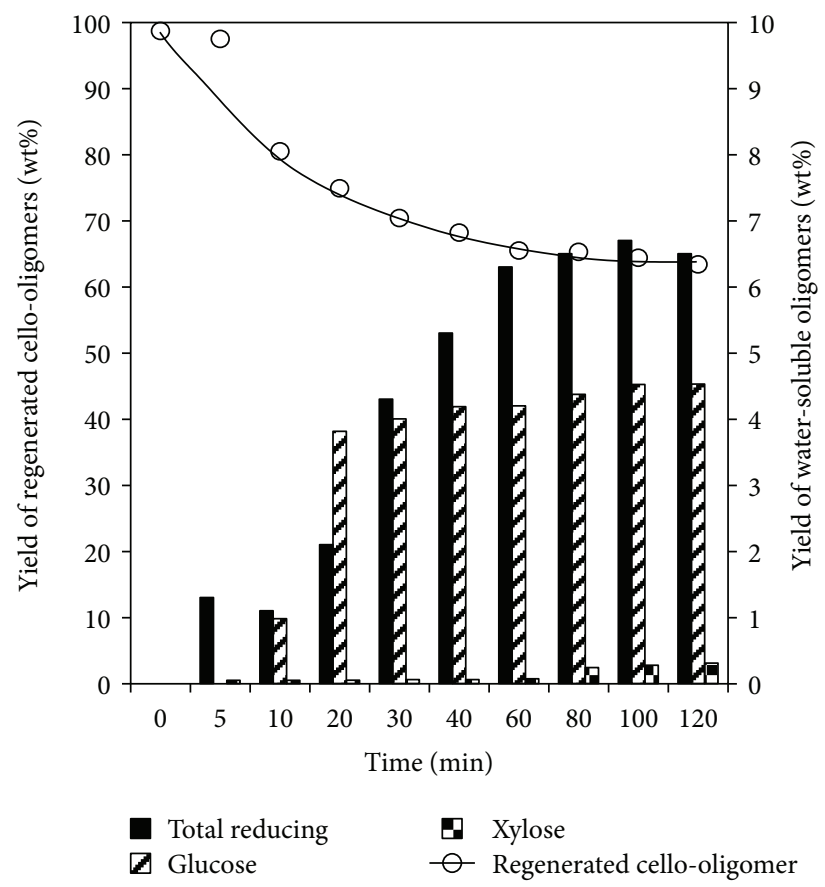

(c)

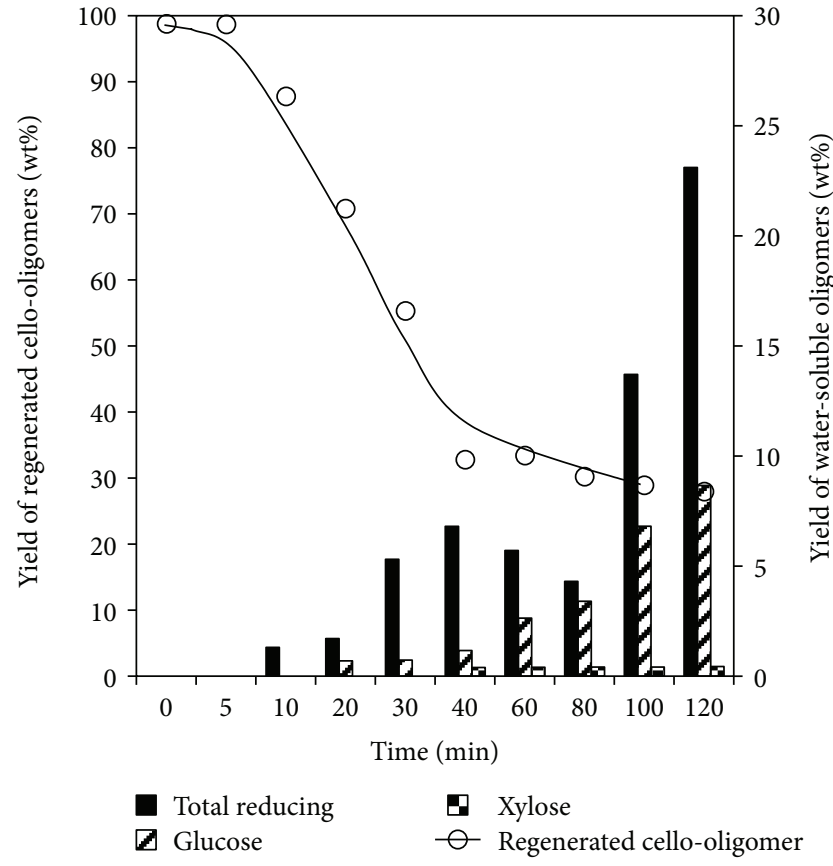

(b)

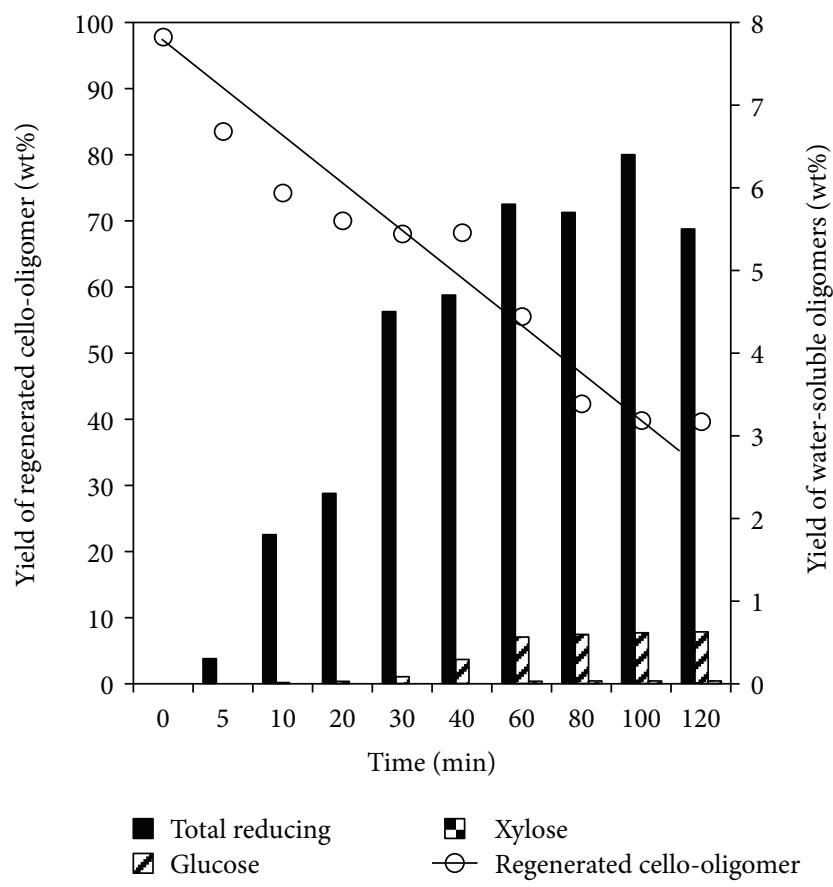

(d)

Figure 4: Yields of cello-oligomers and water-soluble sugar products derived from depolymerization of cellulose fibres of (a) sawdust, (b) sugarcane bagasse, (c) corn cob, and (d) sago pith wastes at different reaction times (acid catalyst concentration $=0.23 \mathrm{mmol}$ $\mathrm{H}_{3} \mathrm{O}^{+}$; temperature $=80^{\circ} \mathrm{C}$ ).

shift continuously albeit more rapidly during the intial stage of depolymerization. Correspondingly, the PDI values of cello-oligomers were observed to decrease comparatively more rapidly from 22.4 to 3.02 within the duration of depolymerization.

It is noteworthy that cellulose fibres isolated from both sago pith waste and sugarcane bagasse samples showed comparatively higher initial mean DPw values of 564 and 571 , respectively. The depolymerization of such cellulose fibres could have occurred in a controlled fashion with a size-specific preference for the cleavage of longer chains. Hence, cello-oligomers with comparatively lower mean $\mathrm{DPw}$ values of 30 and 37, respectively, were obtained after depolymerization for $120 \mathrm{~min}$. However, the lowest PDI 


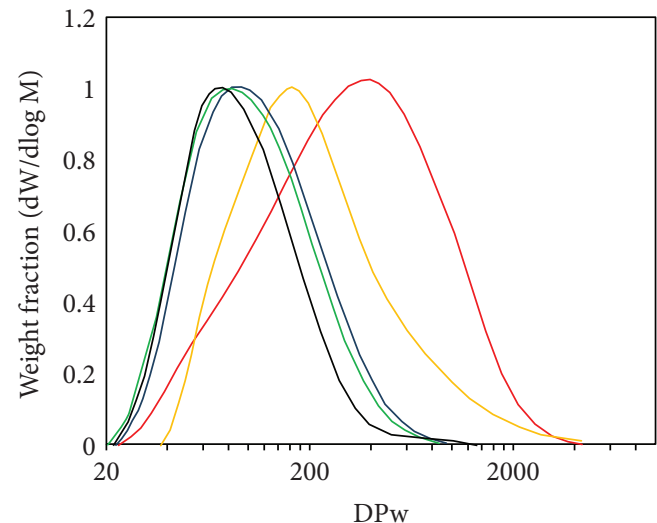

(a)

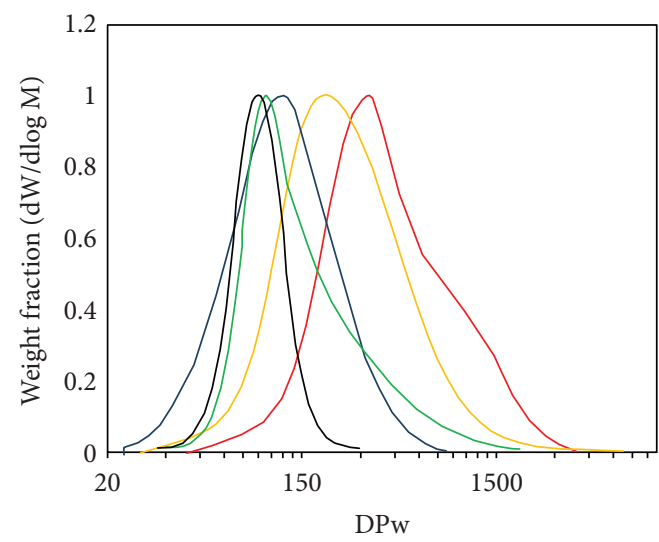

(c)

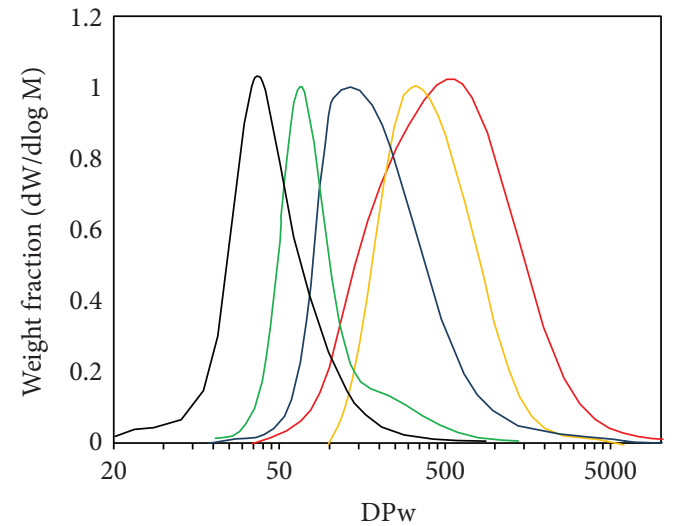

(b)

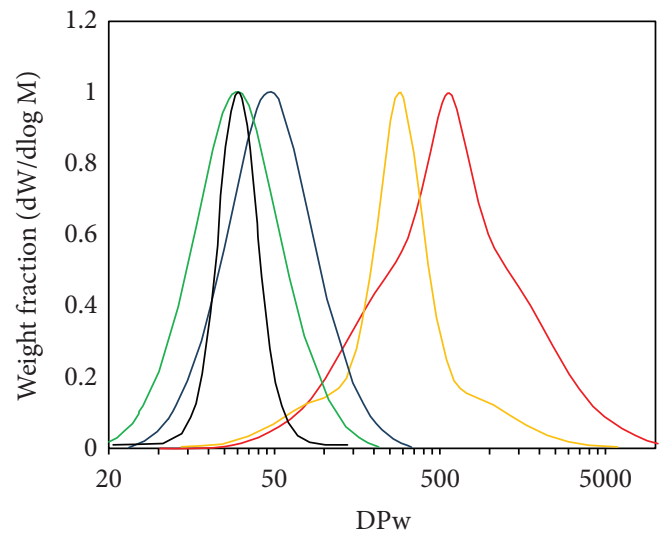

(d)

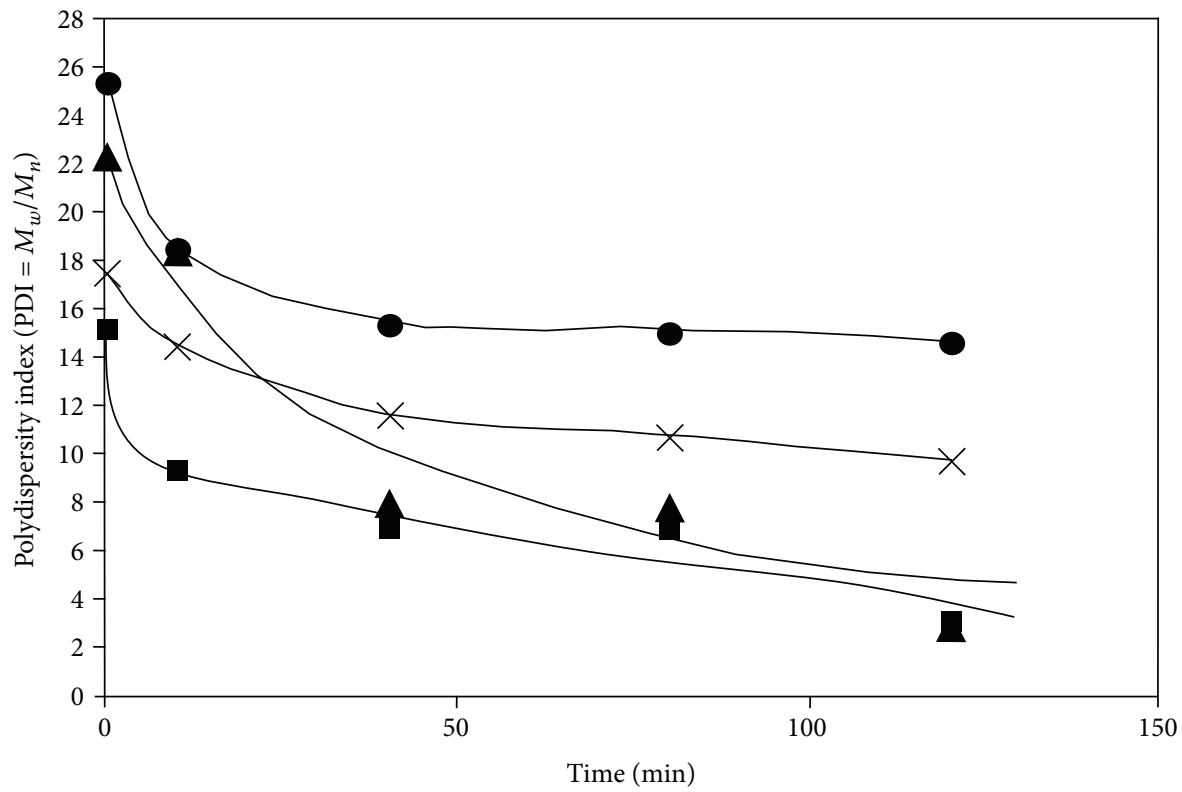

Sawdust $*$ Sugarcane bagasse
Corn cob

$\Delta$ Sago pith waste

(e)

FIGURE 5: DPw distribution of various LBW of the cello-oligomers obtained from cellulose fibres at different reaction times: (a) sawdust, (b) sugarcane bagasse, (c) corn cob, and (d) sago pith wastes and (e) polydispersity index (PDI). (Red line: original cellulose fibres, yellow line: $10 \mathrm{~min}$, blue line: $40 \mathrm{~min}$, green line: $80 \mathrm{~min}$, and black line: $120 \mathrm{~min}$ ). 
TABLE 1: Mean DPw peak values and polydispersity indices (PDI) for cello-oligomers derived from various types of lignocellulosic biomass wastes.

\begin{tabular}{lcccc}
\hline Source of cellulose fibres & \multicolumn{2}{c}{ Mean DPw (peak) } & $\begin{array}{c}\text { Percentage change of mean DPw } \\
\Delta \text { DPw (\%) }\end{array}$ & $\begin{array}{c}\text { Polydispersity index (PDI) } \\
M_{w} / M_{n}\end{array}$ \\
\hline Sago pith wastes & 564 & 30 & 94.7 & 3.02 \\
Sugarcane bagasse & 571 & 37 & 93.5 & 9.78 \\
Sawdust & 351 & 72 & 79.5 & 14.70 \\
Corn cob & 329 & 88 & 78.1 & 3.21 \\
\hline
\end{tabular}

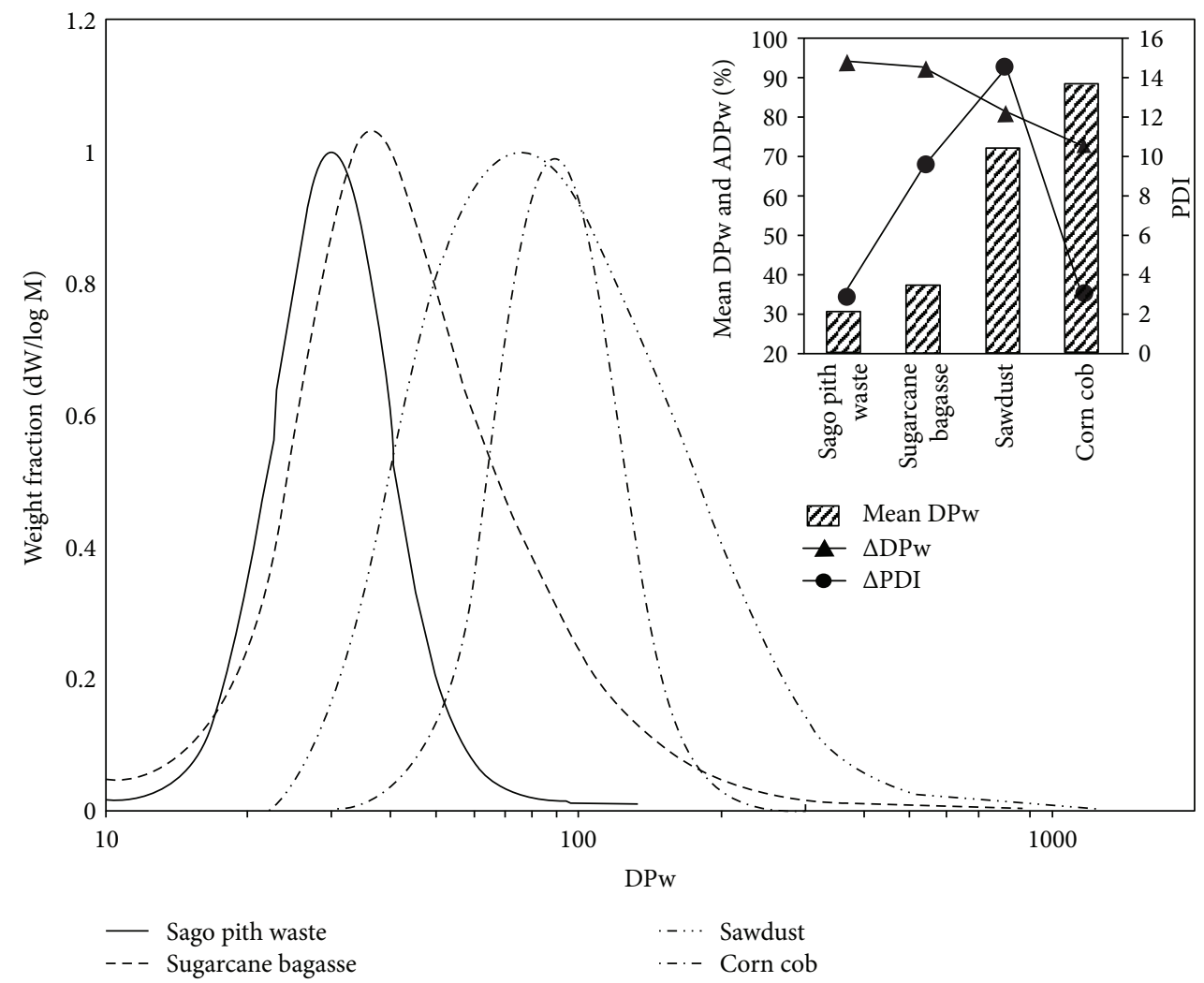

FIGURE 6: DPw distribution of cello-oligomers obtained from cellulose fibres of various LBW after depolymerization for 120 min. Inset shows the mean $\mathrm{DPw}$ value, percentage change of mean $\mathrm{DPw}(\triangle \mathrm{DPw})$ value, and polydispersity indices (PDI) of cello-oligomers.

values for cello-oligomers formed from sago pith waste and sugarcane baggasse samples were 3.02 and 9.78 , respectively (Table 1). The substantial difference in the PDI values could be attributed to the inherent nature and structural differences of cellulose fibres originated from different biomass sources. During depolymerization, hydronium ions from the catalyst enabled the cleavage of longer-chain cellooligomers to produce cello-oligiomers of shorter chain length while cello-oligomers of low $\mathrm{DPw}$ formed watersoluble oligomers.

Figure 6 shows a comparison of the mean DPw distribution of cello-oligomers derived from cellulose fibres of various lignocellulosic biomass wastes after depolymerization for 120 min. The mean DPw peak values of cello-oligomers were observed in the descending order of corn cob, sawdust, sugarcane baggasse, and sago pith waste. In contrast, the width of DPw distributions of cello-oligomers as reflected by their PDI values followed the descending order of sawdust, sugarcane baggasse, corn cob, and sago pith waste (Table 1). Evidently, cello-oligomers derived from cellulose fibres of different biomass sources would possess substantially different $\mathrm{DPw}$ distribution and polydispersity which could in turn affect their physical and chemical properties.

3.5. Depolymerization Kinetics of Cellulose Fibres. The depolymerization kinetics of cellulose fibres derived from various types of lignocellulosic biomass wastes under specific conditions were investigated by applying second-order and pseudo-second-order kinetic equations (Figure 7). Depolymerization of cellulose fibres isolated from sugarcane bagasse and sago pith wastes was observed to follow second-order kinetics, whereas depolymerization of cellulose fibres isolated from sawdust and corn cob exhibited pseudo-second-order kinetics. Differences in the rate constants for the depolymerization of cellulose fibres derived from sawdust $(1.8 \times$ $\left.10^{-4} \mathrm{~min}^{-1}\right)$, corn $\operatorname{cob}\left(1.6 \times 10^{-3} \mathrm{~min}^{-1}\right)$, sago pith waste 


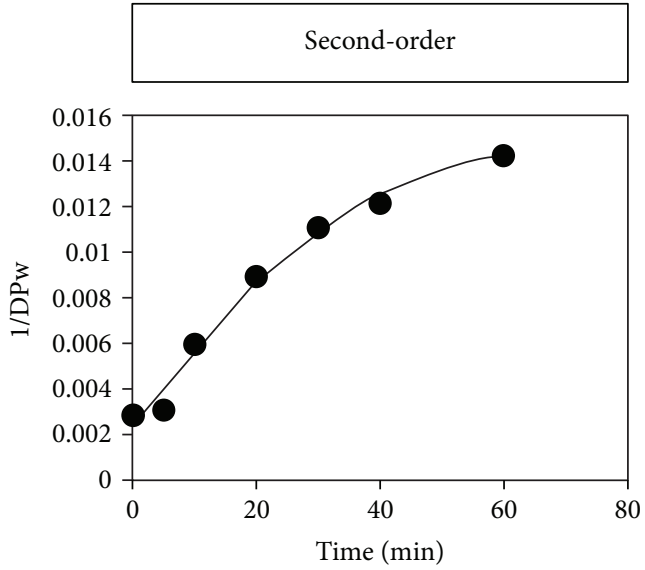

(a)

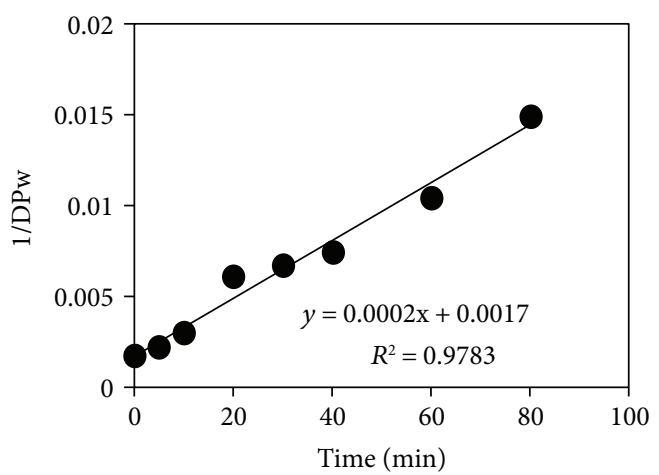

(c)

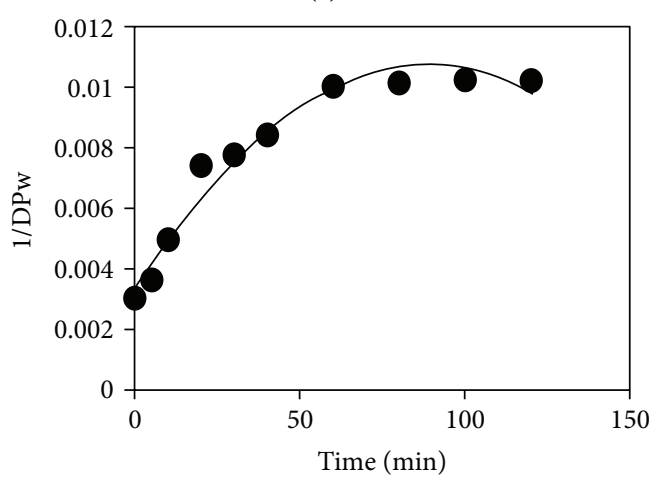

(e)

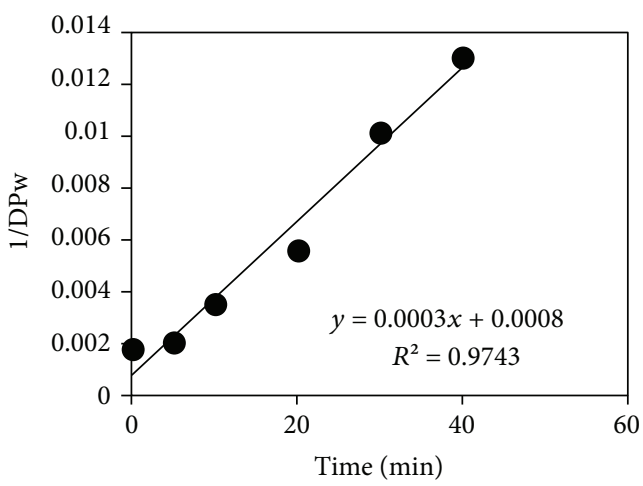

(g)

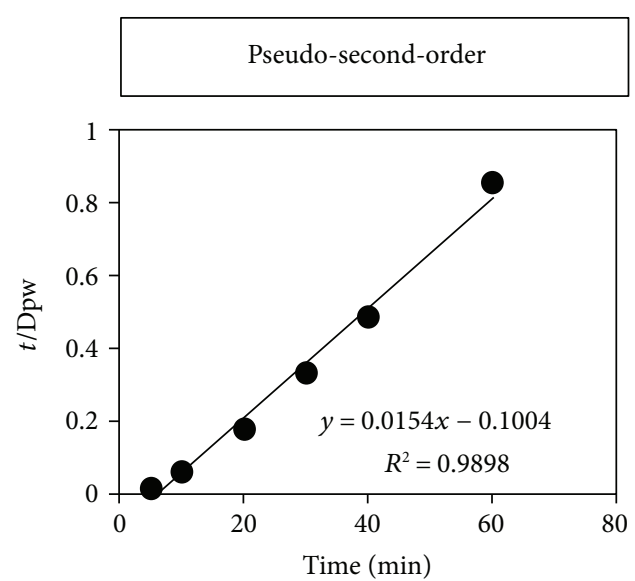

(b)

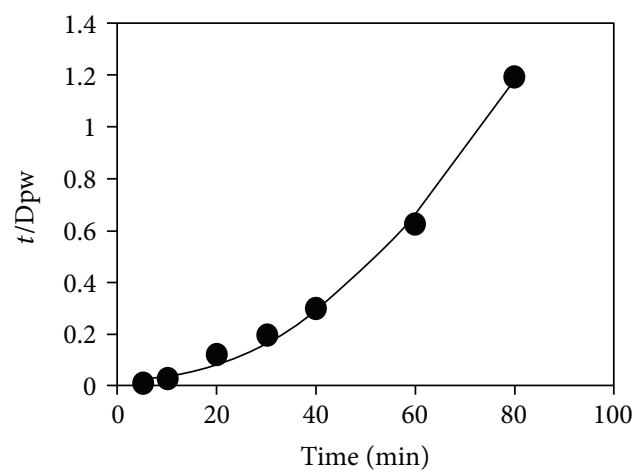

(d)

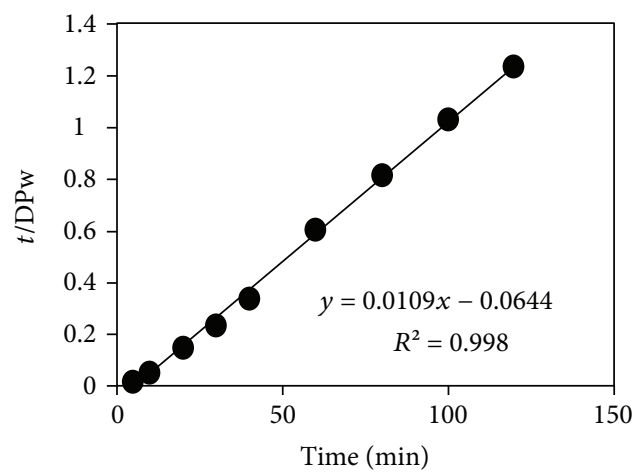

(f)

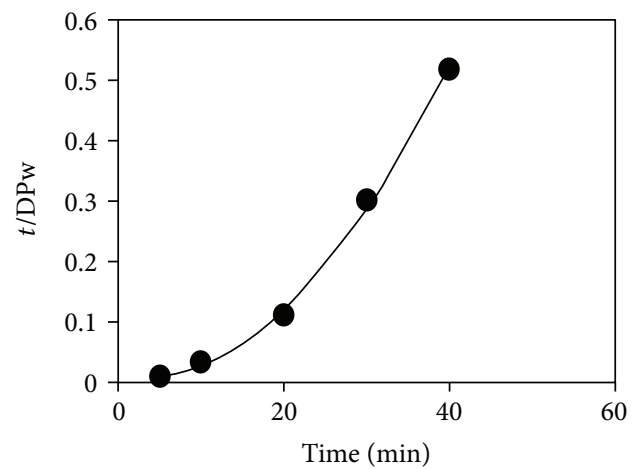

(h)

Figure 7: Second-order and pseudo-second-order kinetics for the depolymerization of cellulose fibres derived from sawdust (a, b), sugarcane bagasse $(c, d)$, corn cob $(e, f)$, and sago pith wastes $(g, h)$. (Reaction temperature $=80^{\circ} \mathrm{C}$; acid catalyst concentration $=0.23 \mathrm{mmol}_{3} \mathrm{O}^{+}$). 
$\left(3 \times 10^{-4} \mathrm{~min}^{-1}\right)$, and sugarcane bagasse $\left(2 \times 10^{-4} \mathrm{~min}^{-1}\right)$ could be due to variations in the composition of crystalline phases within their cellulose fibres. As reported by Cheng et al. [31] and Park et al. [32], the crystalline structure of cellulose molecules could affect the hydrolysis rate of cellulose. These results concurred with findings of our previous study that some degree of control could be afforded on the chain lengths of cello-oligomers formed during the depolymerization of cellulose fibres in ionic liquid and in the presence of a solid catalyst. The rate of depolymerization and mean DPw or chain length of cello-ologomers could be controlled by simply varying the reaction time at a fixed acid catalyst concentration and reaction temperature. Henceforth, cello-oligomers of the most desirable chain length could be tailored in order to optimize physicochemical properties for desired applications, as well as yields of desired final products.

\section{Conclusion}

Cellulose fibres derived from different lignocellulosic biomass wastes such as sawdust, corn cob, sugarcane bagasse, and sago pith wastes were depolymerized under controlled conditions to form cello-oligomers of varying mean $\mathrm{DPw}$ values or chain lengths and polydispersity. Cello-oligomers of mean DPw values less than 100 were obtained from all types of lignocellulosic biomass wastes within a depolymerization duration of $120 \mathrm{~min}$. Cello-oligomers with controllable $\mathrm{DPw}$ values instead of sugars derived from lignocellulosic biomass could be more ideally suited as the renewable feedstock for sustainable biorefineries in the production of biofuels and biobased functional chemicals. Besides, value-added utilization of such agricultural residues or by-products would therefore provide additional revenues for farmers without any adverse impacts on soil fertility and environment.

\section{Data Availability}

Data supporting the results can be found in our recent published article-Pang S.C., Voon L.K., Chin S.F. (2017). Conversion of Sago (Metroxylon sagu) Pith Waste to Fermentable Sugars via a Facile Depolymerization Process. Applied Biochemistry and Biotechnology, DOI 10.1007/ s12010-017-2616-z; Published online 30 September, 2017, or from the corresponding author upon request.

\section{Conflicts of Interest}

The authors do not have any conflicts of interest.

\section{Acknowledgements}

The authors wish to acknowledge the financial support rendered by the Malaysian Ministry of Higher Education (MOHE) via the award of fundamental research grants (Grant nos. FRGS/ST01(01)/967/2013(08) and F07/FRGS/ $1495 / 2016)$, as well as research management and support provided by the Research Innovation and Management Centre (RIMC), Universiti Malaysia Sarawak.

\section{References}

[1] H. Rasmussen, H. R. Sørensen, and A. S. Meyer, "Formation of degradation compounds from lignocellulosic biomass in the biorefinery: sugar reaction mechanisms," Carbohydrate Research, vol. 385, pp. 45-57, 2014.

[2] D. P. Ho, H. H. Ngo, and W. Guo, "A mini review on renewable sources for biofuel,” Bioresource Technology, vol. 169, pp. 742-749, 2014.

[3] R. A. Sheldon, "Green and sustainable manufacture of chemicals from biomass: state of the art," Green Chemistry, vol. 16, no. 3, pp. 950-963, 2014.

[4] H. Zhao, C. L. Jones, G. A. Baker, S. Xia, O. Olubajo, and V. N. Person, "Regenerating cellulose from ionic liquids for an accelerated enzymatic hydrolysis," Journal of Biotechnology, vol. 139, no. 1, pp. 47-54, 2009.

[5] R. Rinaldi, N. Meine, J. vom Stein, R. Palkovits, and F. Schüth, "Which controls the depolymerization of cellulose in ionic liquids: the solid acid catalyst or cellulose ?," Chemistry Sustainable Materials and Energy, vol. 3, pp. 266-276, 2010.

[6] M. Yabushita, H. Kobayashi, and A. Fukuoka, "Catalytic transformation of cellulose into platform chemicals," Applied Catalysis B: Environmental, vol. 145, pp. 1-9, 2014.

[7] R. Rinaldi and F. Schüth, "Acid hydrolysis of cellulose as the entry point into biorefinery schemes," Chemistry Sustainable Materials and Energy, vol. 2, pp. 1096-1107, 2009.

[8] N. V. Plechkova and K. R. Seddon, "Applications of ionic liquids in the chemical industry," Chemical Society Reviews, vol. 37, no. 1, pp. 123-150, 2008.

[9] R. D. Rogers, K. R. Seddon, and S. Volkov, Eds., "Green industrial applications of ionic liquids," Springer Science \& Business Media, vol. 92, pp. 456-478, 2012.

[10] J. Wilkes, "Properties of ionic liquid solvents for catalysis," Journal of Molecular Catalysis A: Chemical, vol. 214, no. 1, pp. 11-17, 2004.

[11] H. Olivier-Bourbigou, L. Magna, and D. Morvan, "Ionic liquids and catalysis: recent progress from knowledge to applications," Applied Catalysis A: General, vol. 373, no. 12, pp. 1-56, 2010.

[12] A. Brandt, J. Gräsvik, J. P. Hallett, and T. Welton, "Deconstruction of lignocellulosic biomass with ionic liquids," Green Chemistry, vol. 15, no. 3, pp. 550-583, 2013.

[13] W. Z. Li, M. T. Ju, Y. N. Wang, L. Liu, and Y. Jiang, "Separation and recovery of cellulose from Zoysia japonica by 1-allyl-3methylimidazolium chloride," Carbohydrate Polymers, vol. 92, no. 1, pp. 228-235, 2013.

[14] I. Van zandvoort, Y. Wang, C. B. Rasrendra et al., "Formation, molecular structure, and morphology of humins in biomass conversion: influence of feedstock and processing conditions," Chemistry Sustainable Materials and Energy, vol. 6, pp. 17451758, 2013.

[15] R. Rinaldi, R. Palkovits, and F. Schüth, "Depolymerization of cellulose using solid catalysts in ionic liquids," Angewandte Chemie International Edition, vol. 47, no. 42, pp. 8047-8050, 2008.

[16] L. K. Voon, S. C. Pang, and S. F. Chin, "Regeneration of cellooligomers via selective depolymerization of cellulose fibers 
derived from printed paper wastes," Carbohydrate Polymers, vol. 142, pp. 31-37, 2016.

[17] Y. Sun and J. Cheng, "Hydrolysis of lignocellulosic materials for ethanol production: a review," Bioresource Technology, vol. 83, no. 1, pp. 1-11, 2002.

[18] H. Zhang, J. Wu, J. Zhang, and J. He, "1-Allyl-3-methylimidazolium chloride room temperature ionic liquid: a new and powerful nonderivatizing solvent for cellulose," Macromolecules, vol. 38, no. 20, pp. 8272-8277, 2005.

[19] F. Kačík, D. Kačíková, M. Jablonský, and S. Katuščák, "Cellulose degradation in newsprint paper ageing," Polymer Degradation and Stability, vol. 94, no. 9, pp. 1509-1514, 2009.

[20] F. Liu, R. K. Kamat, I. Noshadi et al., "Depolymerization of crystalline cellulose catalyzed by acidic ionic liquids grafted onto sponge-like nanoporous polymers," Chemical Communications, vol. 49, no. 76, pp. 8456-8458, 2013.

[21] K. J. Vetter, Electrochemical Kinetics: Theoretical Aspects, Elsevier, New York, NY, USA, 2013.

[22] D. Robati, "Pseudo-second-order kinetic equations for modeling adsorption systems for removal of lead ions using multi-walled carbon nanotube," Journal of Nanostructure in Chemistry, vol. 3, no. 1, p. 55, 2013.

[23] M. Ibrahim, A. Nada, and D. E. Kamal, "Density functional theory and FTIR spectroscopic study of carboxyl group," Indian Journal of Pure \& Applied Physics, vol. 43, pp. 911917, 2005.

[24] H. Wang, B. Li, and B. Shi, "Preparation and surface acid-base properties of porous cellulose," BioResources, vol. 3, pp. 3-12, 2007.

[25] B. Bodirlau and C. A. Teaca, "Fourier transform infrared spectroscopy and thermal analysis of lignocelluloses fillers treated with organic anhydrides," Romania Journal Physics, vol. 54, pp. 93-104, 2007.

[26] E. Smidt, K. Böhm, and M. Schwanninger, The Application of FT-IR Spectroscopy in Waste Management, INTECH Open Access Publisher, 2011.

[27] Y. Sun, L. Lin, H. Deng et al., "Structure of bamboo in formic acid," BioResources, vol. 3, pp. 297-315, 2008.

[28] S. Y. Oh, D. I. Yoo, Y. Shin et al., "Crystalline structure analysis of cellulose treated with sodium hydroxide and carbon dioxide by means of X-ray diffraction and FTIR spectroscopy," Carbohydrate Research, vol. 340, no. 15, pp. 23762391, 2005.

[29] W. Chen, H. Yu, Y. Liu, Y. Hai, M. Zhang, and P. Chen, "Isolation and characterization of cellulose nanofibers from four plant cellulose fibers using a chemical-ultrasonic process," Cellulose, vol. 18, no. 2, pp. 433-442, 2011.

[30] M. Sain and S. Panthapulakkal, "Bioprocess preparation of wheat straw fibers and their characterization," Industrial Crops and Products, vol. 23, no. 1, pp. 1-8, 2006.

[31] G. Cheng, P. Varanasi, C. Li et al., "Transition of cellulose crystalline structure and surface morphology of biomass as a function of ionic liquid pretreatment and its relation to enzymatic hydrolysis," Biomacromolecules, vol. 12, no. 4, pp. 933-941, 2011.

[32] S. Park, J. O. Baker, M. E. Himmel, P. A. Parilla, and D. K. Johnson, "Cellulose crystallinity index: measurement techniques and their impact on interpreting cellulase performance," Biotechnology for Biofuels, vol. 3, no. 1, pp. 10-17, 2010. 


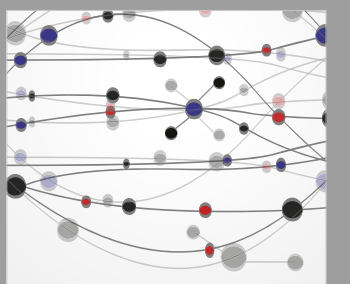

The Scientific World Journal
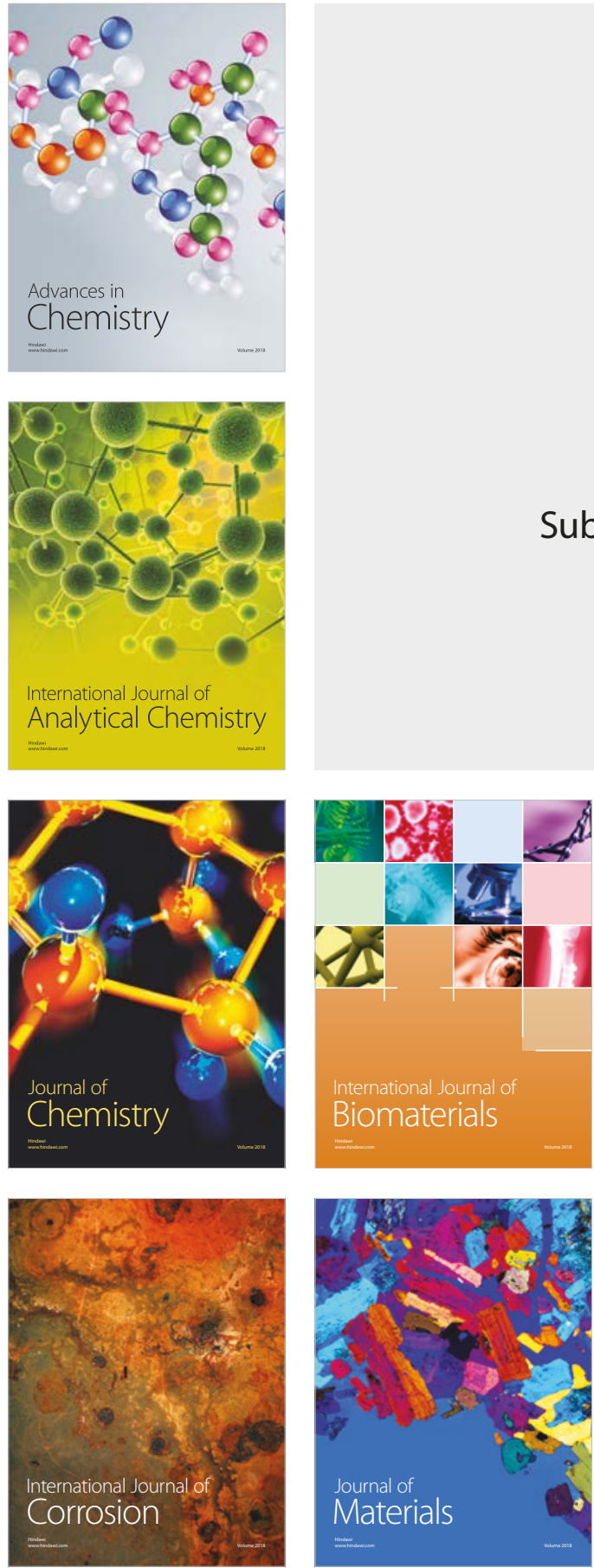

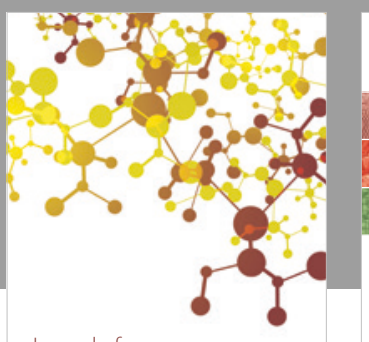

Journal of

Applied Chemistry
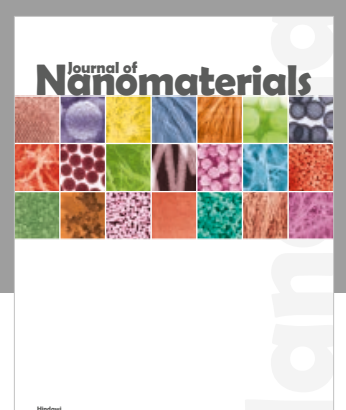

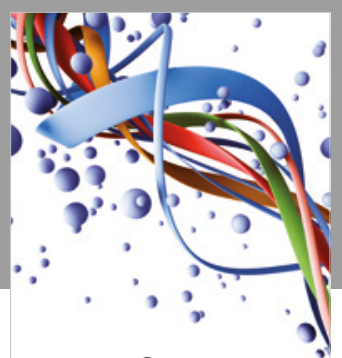

Scientifica

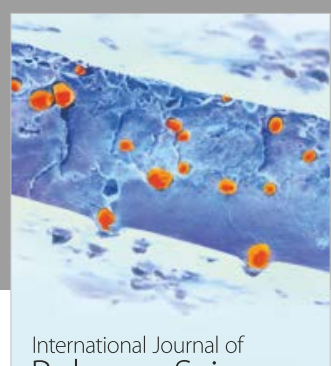

Polymer Science

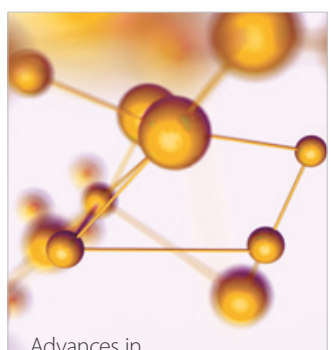

Physical Chemistry
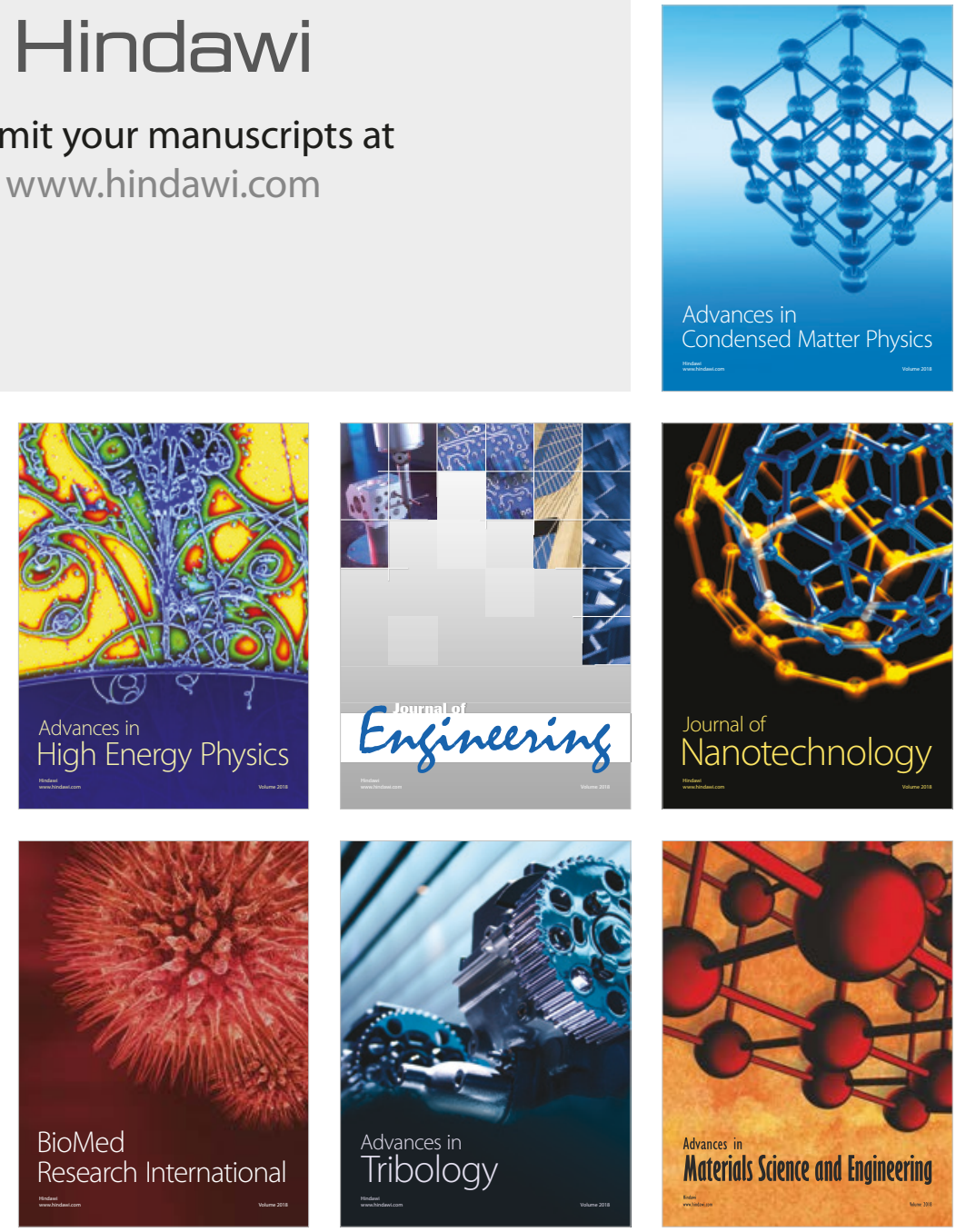